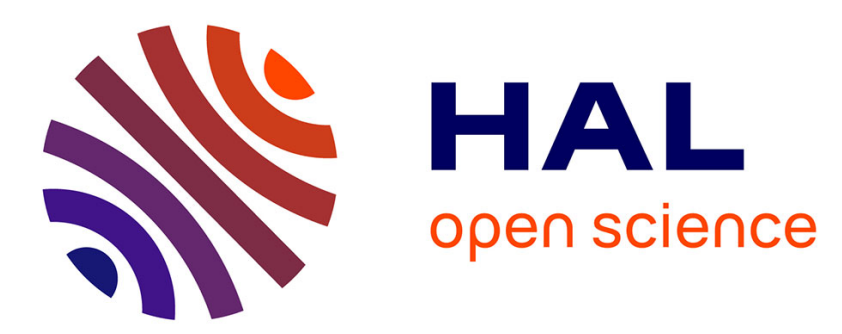

\title{
Absence of bacterial resistance to medical-grade manuka honey
}

R. A. Cooper, L. Jenkins, A. F. M. Henriques, R. S. Duggan, N. F. Burton

\section{To cite this version:}

R. A. Cooper, L. Jenkins, A. F. M. Henriques, R. S. Duggan, N. F. Burton. Absence of bacterial resistance to medical-grade manuka honey. European Journal of Clinical Microbiology and Infectious Diseases, 2010, 29 (10), pp.1237-1241. 10.1007/s10096-010-0992-1 . hal-00600038

\section{HAL Id: hal-00600038 https://hal.science/hal-00600038}

Submitted on 13 Jun 2011

HAL is a multi-disciplinary open access archive for the deposit and dissemination of scientific research documents, whether they are published or not. The documents may come from teaching and research institutions in France or abroad, or from public or private research centers.
L'archive ouverte pluridisciplinaire HAL, est destinée au dépôt et à la diffusion de documents scientifiques de niveau recherche, publiés ou non, émanant des établissements d'enseignement et de recherche français ou étrangers, des laboratoires publics ou privés. 
Diseases

Editorial Manager(tm) for European Journal of Clinical Microbiology \& Infectious

Manuscript Draft

Manuscript Number: EJCMID-D-09-00395R1

Title: Absence of bacterial resistance to medical-grade manuka honey

Article Type: Article

Keywords: manuka honey; resistance; wounds

Corresponding Author: Dr. Professor Rose Cooper, Ph.D

Corresponding Author's Institution: University of Wales Institute Cardiff

First Author: Rose A Cooper, PhD

Order of Authors: Rose A Cooper, PhD; Rose A Cooper, PhD; Leighton Jenkins, BSc; Ana F Henriques, PhD; Sean Duggan, BSc; Rose Cooper, Ph.D; Leighton Jenkins, BSc; Ana F Henriques, PhD; Sean Duggan, BSc; Neil F Burton, PhD

Abstract: Clinical use of honey in the topical treatment of wounds has increased in Europe and North America since licensed wound care products became available in 2004 and 2007 respectively. Honeyresistant bacteria have not been isolated from wounds, but there is a need to investigate whether honey has the potential to select for honey resistance. Two cultures of bacteria from reference collections (Staphylococcus aureus NCTC 10017 and Pseudomonas aeruginosa ATCC 27853) and four cultures isolated from wounds (Escherichia coli, MRSA, Pseudomonas aeruginosa and Staphylococcus epidermidis) were exposed to sub-lethal concentrations of manuka honey in continuous and stepwise training experiments to determine whether susceptibility to honey diminished. Reduced susceptibilities to manuka honey in the test organisms during long-term stepwise resistance training were found, but these changes were not permanent and honey-resistant mutants were not detected. The risk of bacteria acquiring resistance to honey will be low if high concentrations are maintained clinically.

Response to Reviewers: Dear Editor, Thank you for the reviewers' comments on our paper entitled "Absence of bacterial resistance to medical-grade manuka honey". We were pleased that they liked the design and execution of our study and we understood the reservations that reviewer had about our conclusions. We have made the following changes in response to each specific observation, which we feel strengthen our paper: Reviewer 1.

We have inserted line numbers into the paper.

In order to rebut the reservations about our conclusions we have recently performed further tests on the four clinical isolates that were collected at the end of the recovery period and stored at $-80^{\circ} \mathrm{C}$. For each of the thawed cultures, MICs and MBCs were determined in duplicate on three separate occasions. We found that three cultures (P.aeruginosa, S. epidermidis and MRSA) had returned to pre-training levels of susceptibility and that the MIC of E. coli was only 1.4 times higher than at time 0 . That information is included as Table 2 (line 370) and described in lines 201 to 209 . We respectfully maintain that honey-resistant mutants were not recovered. To support this conclusion as advised we noted (lines 241-243) that antibiotic-resistance training led to MICs that increased by factors of either 32 or 64 (citation 17-Blair et al, 2009), while honey resistance training led to an increase of 1.4. We 
also used the EUCAST definition of clinical resistance to show that honey susceptibility which had increased by a factor of 1.4 is unlikely to lead to therapeutic failure when wound care products normally contain at least 80\% manuka honey and normally 95\%(w/v) (lines 258 to 260).

We have removed the statement "gradually increased towards pre-treatment levels".

We have made clear the proportional changes in MICs (lines 195 to 197 and 208 and 251). We have commented on the need to maintain high concentrations of manuka honey in wounds to avoid the selection of resistant strains (lines 278 to 284).

We have modified the Abstract (lines 42 to 45 ) to explain that reduced susceptibility was found during long-term training and we have commented on the need to maintain high concentrations of manuka honey during clinical use (lines 45 to 46 ). In the concluding paragraph we also note that prolonged exposure to antimicrobial agents should be avoided.

We have deleted statements about the development of antibiotic resistance from the Introduction and the Discussion.

We have inserted "preceding days' culture" into line 169, and "day" into line 194.

We have removed the reference to high sugar content, low water content and acidity.

We have changed the captions to Figure 1 and 2.

Reviewer 2.

We were encouraged that our conclusions were considered to be robust.

We have changed MIC against manuka honey to "MICs to manuka honey" throughout the paper.

We have clarified which honey was used in which experiments by changing "preliminary" to "shortterm"(line 91) and explained the activity associated with each honey (lines 100 to 103).

We have inserted "preceding days' culture" in line 133.

In order to clarify the number of MIC tests performed and the experimental design we have inserted a brief explanation in lines 210 to 217.

We hope that you will now be able to accept our manuscript for publication,

Yours sincerely,

Rose Cooper 


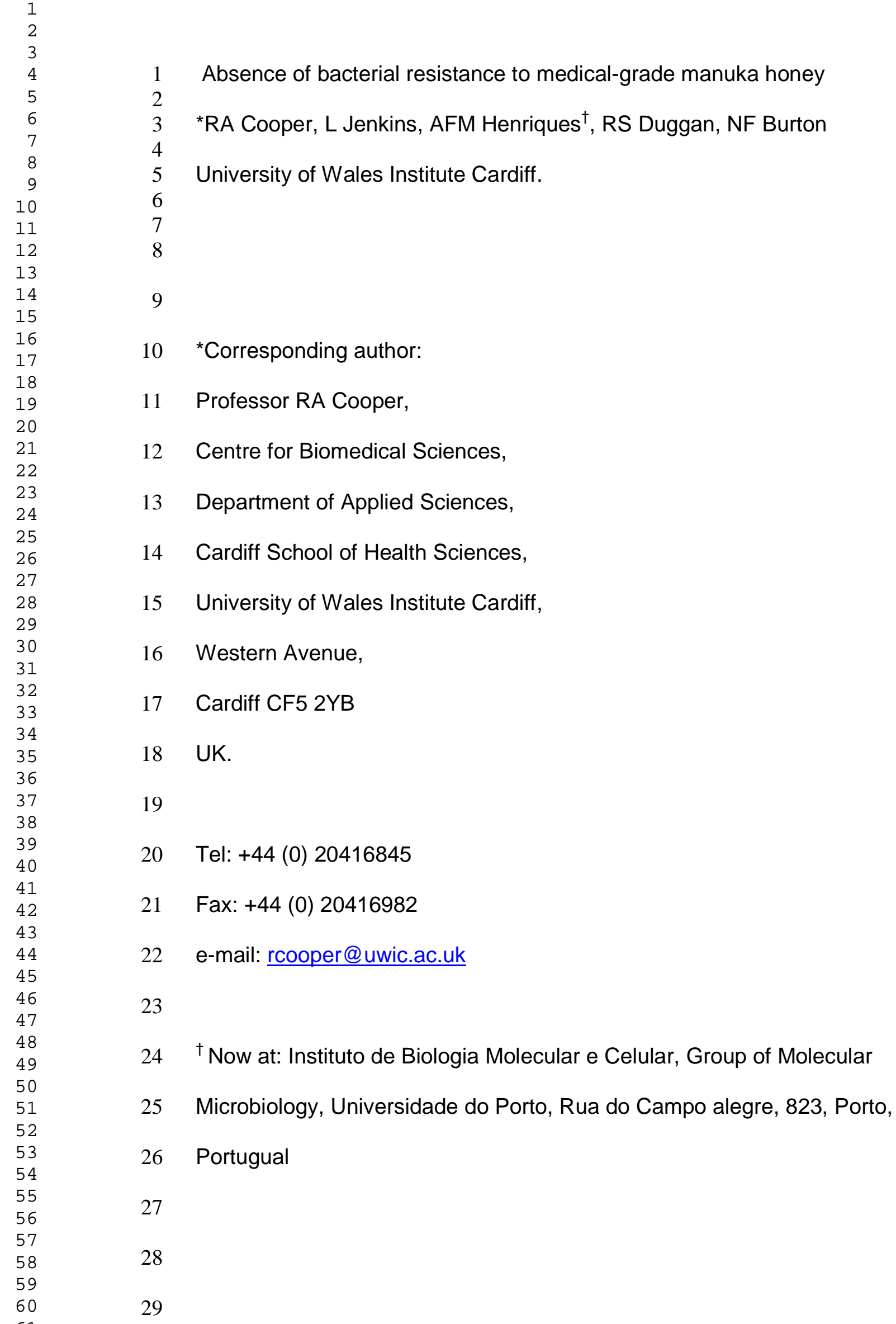$$
3
$$$$
4
$$$$
5
$$$$
6
$$$$
7
$$$$
8
$$

9

$10{ }^{*}$ Corresponding author:

11 Professor RA Cooper,

12 Centre for Biomedical Sciences,

13 Department of Applied Sciences,

14 Cardiff School of Health Sciences,

15 University of Wales Institute Cardiff,

16 Western Avenue,

17 Cardiff CF5 2YB

18 UK.

19

20 Tel: $+44(0) 20416845$

21 Fax: $+44(0) 20416982$

22 e-mail: rcooper@uwic.ac.uk

$24{ }^{\dagger}$ Now at: Instituto de Biologia Molecular e Celular, Group of Molecular

25 Microbiology, Universidade do Porto, Rua do Campo alegre, 823, Porto,

26 Portugual

Absence of bacterial resistance to medical-grade manuka honey

${ }^{*}$ RA Cooper, L Jenkins, AFM Henriques ${ }^{\dagger}$, RS Duggan, NF Burton

University of Wales Institute Cardiff. 
$31 \quad$ Abstract

32 Clinical use of honey in the topical treatment of wounds has increased in

33 Europe and North America since licensed wound care products became

34 available in 2004 and 2007 respectively. Honey-resistant bacteria have not

35 been isolated from wounds, but there is a need to investigate whether honey

36 has the potential to select for honey resistance. Two cultures of bacteria from

37 reference collections (Staphylococcus aureus NCTC 10017 and

38 Pseudomonas aeruginosa ATCC 27853) and four cultures isolated from

39 wounds (Escherichia coli, MRSA, Pseudomonas aeruginosa and

40 Staphylococcus epidermidis) were exposed to sub-lethal concentrations of

41 manuka honey in continuous and stepwise training experiments to determine

42 whether susceptibility to honey diminished. Reduced susceptibilities to

43 manuka honey in the test organisms during long-term stepwise resistance

44 training were found, but these changes were not permanent and honey-

45 resistant mutants were not detected. The risk of bacteria acquiring resistance

46 to honey will be low if high concentrations are maintained clinically.

47 Introduction

48 For thousands of years wounds were treated topically with relatively poorly

49 characterised preparations of antimicrobial agents derived from animal,

50 vegetable and mineral sources [1]. Apart from honey, many of those

51 substances were too toxic for internal use [2]. During the nineteenth century,

52 the chemical industry yielded a variety of antiseptic compounds that were

53 utilised for preventing and treating wound infections [3], but in recent times

54 cytotoxicity has limited their acceptance [4]. A systematic strategy of 
discovering and evaluating promising chemotherapeutic antimicrobial agents was initiated by Paul Ehrlich at the beginning of the twentieth century; it paved the way for the advent of antibiotics, which revolutionised the treatment of infection. These antimicrobial agents with their specific mechanisms of inhibition exhibited selective toxicity against microbial species, rather than patients and were initially effective in controlling infections. Although not universally susceptible to all antibiotics, most microbial species were susceptible to at least one type of antibiotic. To date seventeen classes of antibiotic have been introduced, but the rate of discovery of new antibiotics has slowed within the past twenty years. The therapeutic value of many antibiotics has been curtailed by the acquisition of resistance in species that were once susceptible, as well as the emergence of species with resistance to multiple antibiotics. This is a real concern because species resistant to every known antibiotic have emerged [5], antiseptic resistant strains have been isolated and organisms with both antibiotic and antiseptic resistance determinants have been discovered [6]. The speed of emergence of antimicrobial resistance is unpredictable [7], but whenever a new inhibitory agent is introduced, resistant organisms emerge in due course. With fewer effective antimicrobial agents to manage wound infections, impacts such as increased morbidity, treatment costs and mortalities have ensued [8]. Novel antimicrobial remedies are needed, but there is always the possibility that organisms with resistance to these agents will arise. 
80 extended to Canada, Europe, Hong Kong, New Zealand and United States of

81 America. As with antibiotics, extensive use of honey may well provide a

82 selective pressure for the emergence of honey resistant strains of wound

83 pathogens. This can be tested in vitro by either repeated cultivation of

84 bacteria in a sub-lethal concentration of the agent, or by incubation in

85 stepwise increasing concentrations. The aim of this study, therefore, was to

86 determine whether bacteria continuously exposed to low concentrations of

87 manuka honey changed their susceptibility to manuka honey and whether

88 resistant strains were selected.

\section{Methods}

90 Organisms tested

91 In short-term resistance training experiments Staphylococcus aureus NCTC

9210017 and Pseudomonas aeruginosa ATCC 27853 were utilised with a

93 sample of sterile manuka honey provided by the New Zealand Natural Food

94 Company. For the rest of the study clinical isolates of MRSA, Staphylococcus

95 epidermidis, Escherichia coli and Pseudomonas aeruginosa were tested with

96 a sample of manuka honey that was provided by Comvita UK (Manukacare

97 18+). This product is available as Medihoney in Canada and the United

98 States. The clinical isolates were cultures of bacteria that had been isolated

99 from patients with chronic wounds attending a local out-patient clinic at

100 University Hospital of Wales, Cardiff. Using a bioassay [9], the antibacterial

101 activity of New Zealand Natural Food company honey sample was found to be

102 equivalent to $19 \%(w / v)$ phenol and Manukacare $18+$ was equivalent to

$10318 \%(w / v)$ phenol.

104 Determination of Minimum Inhibitory Concentrations (MICs) 
105 MICs were determined in 96 well, flat bottomed microtitre plates (Nunc,

106 Roskilde, Denmark) using manuka honey dissolved in either nutrient broth

107 (NB; Oxoid, Basingstoke, UK) or isosensitest broth (ISB; Oxoid, Basingstoke,

108 UK). Honey concentrations varying by $1 \%(w / v)$ were used in successive wells

109 and all wells contained $200 \mu$ l. Wells were inoculated with $1 \mu$ undiluted

110 overnight broth cultures of test organisms (approximately $1 \times 10^{6} \mathrm{cfu} / \mathrm{ml}$ ) and

111 incubated at $37^{\circ} \mathrm{C}$ for $24 \mathrm{~h}$. Positive (broth and inoculum) and negative

112 controls (broth and honey) were included. MICs were determined visually and

113 confirmed by measuring absorbance at $400 \mathrm{~nm}$ in a microtitre plate reader

114 (Anthos Labtec Instruments).

115 Short-term resistance training by exposure to a single sub-lethal_concentration

116 of manuka honey.

117 To one $100 \mathrm{ml}$ conical flask with $20 \mathrm{ml} \mathrm{NB}$ containing $2.5 \%(\mathrm{w} / \mathrm{v})$ manuka

118 honey, $40 \mu \mathrm{l}$ overnight broth culture of $S$. aureus was inoculated and to

119 another $100 \mathrm{ml}$ conical flask with $20 \mathrm{ml} \mathrm{NB}$ containing $8 \%(\mathrm{w} / \mathrm{v})$ manuka

120 honey, $40 \mu \mathrm{l} P$. aeruginosa was inoculated. Both flasks were incubated at

$1213^{\circ} \mathrm{C}$ in a shaking water bath (100 cycles per min) for $24 \mathrm{~h}$. On 10 successive

122 days similar, freshly prepared flasks were inoculated with $40 \mu \mathrm{l}$ from each

123 preceding days' culture, respectively. MICs for each test organism were

124 determined on days 0 and 10 and compared by the Mann-Whitney test using

125 Minitab version 14

126 Short-term resistance training by exposure to stepwise increasing

127 concentrations of manuka honey.

128 Cultures were set up as above using an initial concentration of $1 \%(\mathrm{w} / \mathrm{v})$

129 manuka honey in NB for $S$. aureus and 5\%(w/v) manuka honey in NB for $P$. 
aeruginosa and incubated at $3^{\circ} \mathrm{C}$ with shaking for $24 \mathrm{~h}$. At $24 \mathrm{~h}$ intervals on

13110 consecutive days, freshly prepared broths with honey concentrations

132 increased by $0.5 \%(\mathrm{w} / \mathrm{v})$ for $S$. aureus and $1 \%(\mathrm{w} / \mathrm{v})$ for $P$. aeruginosa were

133 inoculated with $40 \mu \mathrm{l}$ from the preceding days' culture. Throughout the

134 training period the identity of each culture was confirmed daily by BBL Crystal

135 kits (Becton Dickinson, Basingstoke, UK). For both test organisms MICs of

136 manuka honey were determined before and after the training period.

137 Stepwise resistance training of clinical isolates by long-term exposure to sub-

138 inhibitory concentrations of manuka honey.

139 MICs of manuka honey for each of the clinical isolates were determined

140 before continuous exposure to honey. In a 96 well, flat bottomed microtitre

141 plate for each of the 4 test organisms a range of 15 concentrations of manuka

142 honey (Comvita manukacare $18+$ ) in ISB that varied by $1 \%(w / v)$ intervals and

143 included concentrations above and below the initial MIC of each test organism

144 was prepared. Each well contained $200 \mu \mathrm{L}$ ISB and honey and was inoculated

145 on day 0 with $5 \mu$ l overnight broth culture, then incubated at $37^{\circ} \mathrm{C}$ for $24 \mathrm{~h}$.

146 MIC was determined visually as the lowest concentration of honey to prevent

147 growth. A fresh microtitre plate was prepared every $24 \mathrm{~h}$ with a new range of

14815 honey concentrations contained in ISB that increased by $1 \%(\mathrm{w} / \mathrm{v})$

149 increments and included the last MIC in the middle of the range. With time,

150 increasingly higher concentrations of honey in ISB were required, but the

151 range always included honey concentrations above and below the expected

152 MIC. On each successive day of the experiment, wells in the new microtitre

153 plate were inoculated with $5 \mu \mathrm{l}$ of each respective test organism taken from

154 the well of the previous microtitre plate containing the highest concentration of 
155 honey with viable cells (i.e. one well below the actual MIC). Also, the purity of

156 each inoculum was checked by dropping $20 \mu$ l onto nutrient agar plates and

157 streaking out to achieve isolated colonies after incubation at $37^{\circ} \mathrm{C}$ for 24

158 hours. The experiment was continued for 28 successive days with each test

159 organism using an appropriate range of honey concentrations above and

160 below the last recorded MIC value. Cultures obtained after 28 days

161 continuous exposure to a sub-lethal concentration of honey were frozen and

162 stored at $-80^{\circ} \mathrm{C}$ for future reference.

163 Recovery of cultures of clinical isolates following long-term exposure to 164 honey.

165 Each 'honey-trained' culture obtained after 28 days continuous exposure to 166 manuka honey was inoculated into $20 \mathrm{ml}$ isosensitest broth (ISB) contained

167 within a $100 \mathrm{ml}$ conical flask and incubated in a shaking water bath at $37^{\circ} \mathrm{C}$ for

16824 hours. On each successive day, one of four fresh flasks of ISB were

169 inoculated with $100 \mu \mathrm{l}$ of each culture transferred from the preceding days'

170 culture, respectively until 28 days had elapsed. At each daily transfer MIC

171 against manuka honey was determined. Cultures obtained after the 28 day

172 recovery period were frozen and stored at $-80^{\circ} \mathrm{C}$.

173 Cultural characteristics.

174 Identity of the test organisms before the start of the long-term training regime, 175 collected after the 28 day training period and after the 28 day recovery period

176 was determined by Crystal kits (Becton Dickinson, Basingstoke, UK) and by

177 RAPD typing.

\section{Results}

179 Short-term resistance training with reference cultures. 
180 When S. aureus NCTC 10017 was cultured in a sub-lethal concentration of

181 manuka honey for 10 consecutive days, susceptibility to manuka honey did

182 not decrease significantly (Table 1). Conversely the MIC for $P$. aeruginosa

183 ATCC 27853 increased significantly from 15.7 to $19.7 \%(\mathrm{w} / \mathrm{v})$, indicating that

184 the culture had become less susceptible to manuka honey. However, after 6

185 subcultures in honey free NB, there was no significant difference in MIC

186 compared to the starting value.

187 When these two test organisms were successively cultured in stepwise

188 increasing concentrations of honey, viable bacteria were not recovered above

189 their starting MIC values. Hence honey resistance was not observed in either

190 S. aureus or P.aeruginosa.

192 Resistance training of clinical isolates by long-term exposure to honey.

193 The susceptibility of the 4 clinical isolates tested in this study for manuka

194 honey decreased during the 28 day resistance training period (Fig. 1), with the

195 greatest proportional change observed for $E$. coli (2.1) and the least in $P$.

196 aeruginosa (1.4). MICs of MRSA and S. epidermidis increased by factors of

1971.6 and 1.7, respectively . However during the recovery period, MIC values

198 returned to pre-training values within 9 and 14 days for $S$. epidermidis and $P$.

199 aeruginosa respectively, demonstrating that honey-resistant strains of these

200 bacteria had not been selected (Fig. 2). Neither MRSA nor E. coli returned to

201 the pre-training MIC values within the 28 day recovery period. Several months

202 later the cultures frozen at the end of the recovery period were thawed and

203 MICs were determined in duplicate on three separate occasions (Table 2).

204 MBCs were also determined by streaking out samples from microtitre plate 
205 wells showing no growth onto nutrient agar. It was seen that the susceptibility

206 of three of the clinical isolates was similar to those values obtained before

207 resistance training (Fig. 1), but the MIC of manuka honey in E.coli remained

2081.4 times higher. MBC values were close to MICs indicating that manuka

209 honey was bactericidal for the recovered cultures.

210 It is perhaps worth noting that by exposing each test organism to a range of

211 honey concentrations simultaneously during the long-term training

212 experiment, MIC values were obtained daily at each transfer step. This meant

213 that only one determination was obtained at each time point. MICs obtained

214 later on stored cultures were done in replicate (Table 2). Unlike antibiotic

215 testing where serial doubling dilutions series are normally utilised, here honey

216 concentrations differing by $1 \%(\mathrm{w} / \mathrm{v})$ intervals were used to get a more precise

217 estimate of MIC.

218 Purity plates and biochemical characteristics confirmed that culture identities

219 were maintained throughout the training and recovery periods; RAPD profiles

220 remained constant.

\section{Discussion}

222 The excessive use and misuse of antibiotics is known to favour the selection

223 of resistant strains and to inhibit sensitive strains. In Norway, for example,

224 where mupirocin was not available, mupirocin-resistant strains of $S$. aureus

225 were not detected [10]. Restricting mupirocin use in an American medical

226 centre caused an immediate reduction in the occurrence of mupirocin-

227 resistant MRSA [11] and an association between exposure to fusidic acid and

228 fusidic acid-resistance in S.aureus has been established [12]. Resistance to

229 biocides used for skin and wound care has also been demonstrated [6].In the 
230 first 'acclimatization' experiments in 1887, reduced sensitivity to mercuric

231 chloride and boric acid was noted [13]; increased tolerance towards many

232 other antibiotics and antiseptics has since been investigated. Inability to select

233 for resistance to povidone iodine has been reported [13], yet Pseudomonas

234 stutzeri was shown to develop stable resistance to each of chlorhexidine

235 diacetate and cetylpyridinium chlorine when exposed to stepwise increases in

236 concentration [14]. Bacterial resistance to silver, which is often used in wound

237 care, is well defined and debated [15 and16]. Furthermore, short-term

238 stepwise resistance training of $P$. aeruginosa ATCC 27853 to each of

239 tetracycline, ciprofloxacin and honey, and of S.aureus ATCC 9144 to each of

240 tetracycline, oxacillin and honey demonstrated acquisition of antibiotic

241 resistance but not honey resistance [17]. In that study antibiotic-resistant

242 strains recovered after stepwise resistance training to antibiotics exhibited

243 antibiotic susceptibilities significantly reduced by factors of 32 and 64 [17].

244

245 Our study showed that continuous exposure to sub-lethal concentrations of

246 manuka honey for up to 28 days failed to select for honey resistant mutants in

247 any of two reference cultures or four clinical isolates. Reduced susceptibility to

248 manuka honey was observed throughout the long-term training period in each

249 of the clinical isolates. Unlike the large changes in susceptibilities seen in

250 antibiotic resistance training [17], honey MICs increased in P. aeruginosa,

251 MRSA, S. epidermidis and E. coli by factors of 1.4, 1.6, 1.7 and 2.1,

252 respectively (Fig. 1). These changes, however, were not permanent because

253 susceptibility to honey was found to increase during cultivation in honey-free

254 media and during storage at $-80^{\circ} \mathrm{C}$. MICs of final cultures (Fig. 2 and Table 2) 
were close to pre-training MIC values (Fig. 1), except for E. coli which

256

remained 1.4 times higher than at the start of the honey resistance training

257

period. It is not known why susceptibility to honey increased in E.coli and

258

MRSA during storage at $-80^{\circ} \mathrm{C}$. The European consensus of a clinically

259

resistant organism is one in which the level of antimicrobial susceptibility has

260

a high likelihood of clinical failure [18]. Since the concentrations of medical-

261

grade manuka honey that are contained in contemporary licensed wound care

262

products usually exceed $80 \%(\mathrm{w} / \mathrm{v})$ and many are $95 \%(\mathrm{w} / \mathrm{v})$, the honey

263

susceptibilities found in our experiments are not likely to give rise to clinical

264 failure.

265

266 Topical application of honey to wounds will invariably result in dilution,

267 depending on the extent of exudation, and concentrations in vivo must always

268 exceed those shown to be inhibitory in vitro by a significant margin. Providing

269 that the concentration of manuka honey in practice exceeds the highest MBC

270 recorded here by a factor of three, the risk of selecting honey-resistant

271 organisms should be low. Regular dressing changes will help to keep levels of

272 manuka honey high, particularly in highly exuding wounds. Maintaining high

273 levels of antimicrobial agents in the wound environment is always necessary

274 to effectively inhibit viable bacteria before resistant strains emerge. For some

275 biocides cytotoxicity may then become a limitation, but this should not be an

276 issue with manuka honey.

277 Our findings confirm and extend those of Blair and colleagues [17]. With

278 increasing topical use of honey in wounds, the possibility of selecting for

279 honey-resistant wound pathogens is raised. This study suggests that this 
280 outcome will be remote if high concentrations of manuka honey are

281 maintained in clinical practice. Prolonged exposure to antimicrobial agents,

282 including honey, should always be avoided. Hopefully manuka honey will

283 continue to be used as a whole product and specific active components [19-

284 21] will not be purified and used alone, because this is more likely to promote

285 the development of honey-resistant bacteria.

286 Acknowledgements

287 This study was supported in part by Derma Sciences Inc. New Jersey, USA.

288 References

289 1. Forrest RD (1982) Early history of wound treatment. J Roy Soc Med 75:

$290 \quad 198-205$

291 2. Moellering RC (1995) Past, present, and future of antimicrobial agents. The 292 Am J Med 99 (suppl 6A):11S -18S

293 3. Hugo WB (1991) A brief history of heat and chemical preservative and

294 disinfection. J Appl Bacteriol 71: 9-18

295 4. Brennan SS, Leaper D (1985) The effect of antiseptics on the healing

296 wound: a study using the rabbit ear chamber. Br J. Surg 72 (10): 780-782

297 5. Payne DJ, Gwynn MN, Holmes DJ, Pompliano DL (2007) Drugs for bad

298 bugs: confronting the challenges of antibacterial discovery. Nat Rev Drug

299 Discov 6: 29-40

300 6. Russell AD (2002) Introduction of biocides into clinical practice and the 301 impact on antibiotic-resistant bacteria. J Appl Microbiol 92 Suppl: 121S-35S

302 7. Davies J (1996) Bacteria on the rampage. Nature 383: 219-220

303 8. Filius PMG, Gyssens, IC (2002) Impact of increasing antimicrobial

304 resistance on wound management. Am J Clin Dermatol 3: 1-7 
305 9. Allen K, Molan P, Reid G (1991) A survey of the antibacterial activity of some

306 New Zealand honeys. J Pharm Pharmacol 43 (12): 817-882

307 10. Afset JE, Mæland JA (2003) Susceptibility of skin and soft-tissue isolates

308 of Staphylococcus aureus and Streptococcus pyogenes to topical antibiotics.

309 Scand J Infect Dis 35: 84-89

310 11. Walker ES, Levy F, Shorman M, David G, Abdulla J, Sarubbi FA (2004) A

311 decline in mupirocin resistance in methicillin resistance in methicillin-resistant

312 Staphylococcus aureus accompanied administrative control of prescriptions. J

313 Clin Microbiol 42 (6): 342-346

314 12. Mason BW, Howard AJ (2004) Fusidic acid resistance in community

315 isolates of methicillin susceptible Staphylococcus aureus and the use of

316 topical fusidic acid: a retrospective case-controlled study. Int J Antimicrobiol.

317 Agents 23: 300-303

318 13. Houang E, Gilmore OJA, Reid C, Shaw E (1976) Absence of bacterial

319 resistance to povidone iodine. J Clin Path 29: 752-755

320 14. Tattawasart U, Maillard J-Y, Furr JR, Russell AD (1999) Development of

321 resistance to chlorhexidine diacetate and cetylpyridinium chloride in

322 Pseudomonas stutzeri and changes in antibiotic susceptibility. J Hosp Infect

$323 \quad 42: 219-229$

324 15. Silver S, Phung le T, Silver G (2006) Silver as a biocide in burn and

325 wound dressings and bacterial resistance to silver compounds. J Ind Microbiol

326 Biotechnol $33(7): 627-34$

327 16. Percival SL, Bowler PG, Russell D (2005) Bacterial resistance to silver in 328 wound care. J Hosp Infect 60: 1-7 
329 17. Blair SE, Cokcetin NN, Harry EJ, Carter DA (2009) The unusual

330 antibacterial activity of medical-grade Leptospermum honey: antibacterial

331 spectrum, resistance and transcriptome analysis. Eur J Clin Microbiol Doi 10

332 1007/s10096-009-0763-z

333 18. EUCAST (2009) http://www.srga.org/Eucast/eucastdefinitions.htm

334 (accessed on 26th October 2009)

335 19. Molan PC (1992) The antibacterial nature of honey: 1 . The nature of the

336 antibacterial activity. Bee World 73(1): 5-28

337 20. Mavric E, Wittmann S, Barth G, Henle T (2008) Identification and

338 quantification of methylglyoxal as the dominant antibacterial constituent of

339 Manuka (Leptospermum scoparium) honeys from New Zealand. Mol Nutr

$340 \quad$ Food Res $52(4): 483-9$

341 21. Adams CJ, Boult CH, Deadman BJ, et al (2008) Isolation by HPLC and

342 characterization of the bioactive fraction of New Zealand manuka

343 (Leptospermum scoparium) honey. Carbohydrate Res 343: 651-659

344

345

346

347

348

349

350

351

352

353 
354 Table 1: Sensitivity of two reference cultures to manuka honey before and 355 after culture in sub-lethal concentrations of honey for 10 successive days.

\begin{tabular}{|l|c|c|}
\hline \multirow{2}{*}{} & \multicolumn{2}{|c|}{ Mean MIC \%(w/v) \pm sd (n) } \\
\cline { 2 - 3 } & P.aeruginosa ATCC 27853 & S.aureus NCTC 10017 \\
\hline Before training & $15.7 \pm 2.0(8)$ & $2.8 \pm 0.4(5)$ \\
\hline After training & $19.7 \pm 1.4(7)$ & $3.8 \pm 0.08(5)$ \\
\hline$p$ & 0.03 & $>0.05$ \\
\hline
\end{tabular}

\section{7 sd $=$ standard deviation; $n=$ number of assays}

358 Fig.1: Sensitivity of four clinical isolates to manuka honey during exposure to 359 sub-lethal concentrations of manuka honey for 28 days

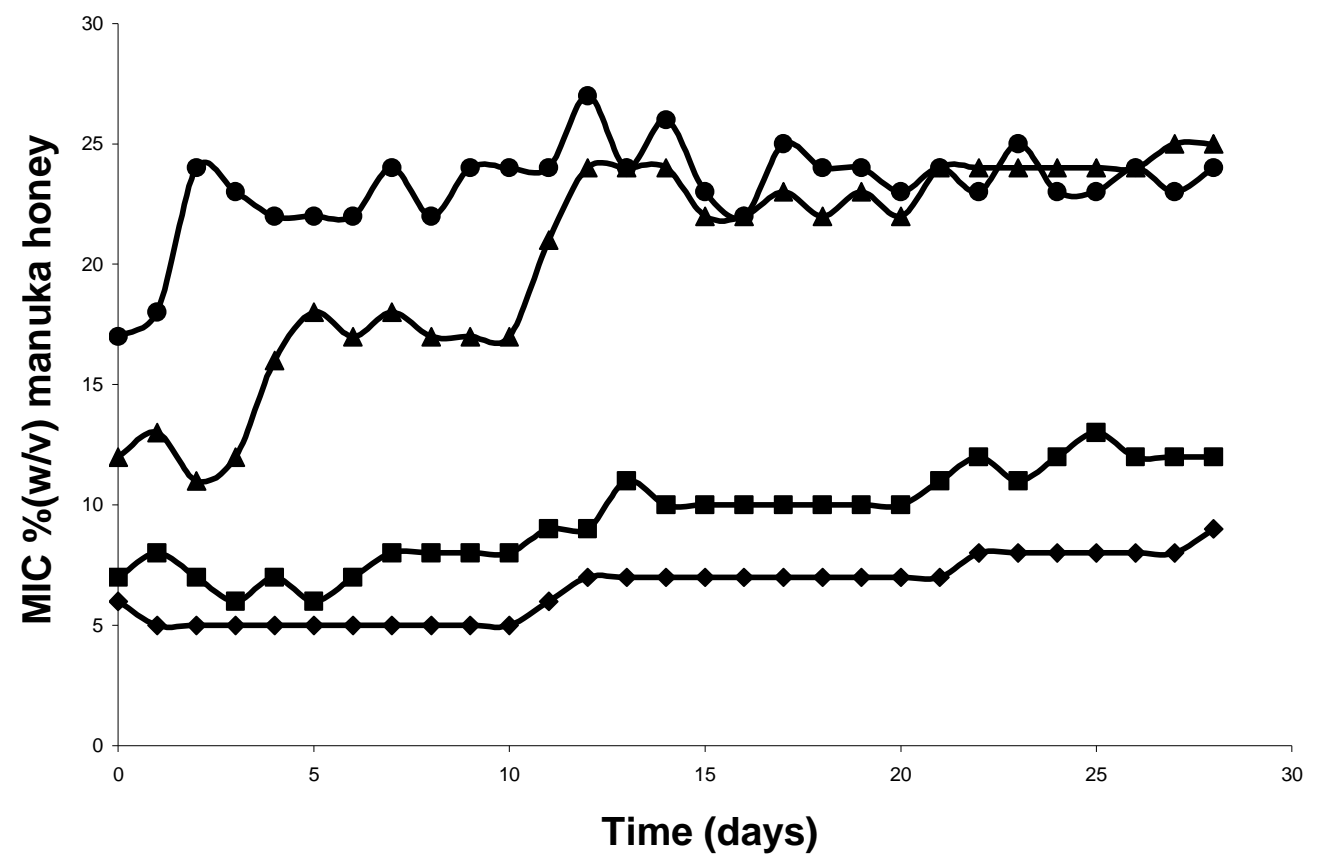

360

361 MICs of manuka honey in subcultures of four clinical isolates during a long362 term honey resistance training experiment. Cultures were isolated from 363 patients with chronic wounds: E. coli (triangles), MRSA (diamonds), $P$. 364 aeruginosa (circles) and S. epidermidis (squares). . 
365 Fig. 2: Sensitivity of four clinical isolates to manuka honey during recovery by 366 cultivation in nutrient broth following long-term honey-training

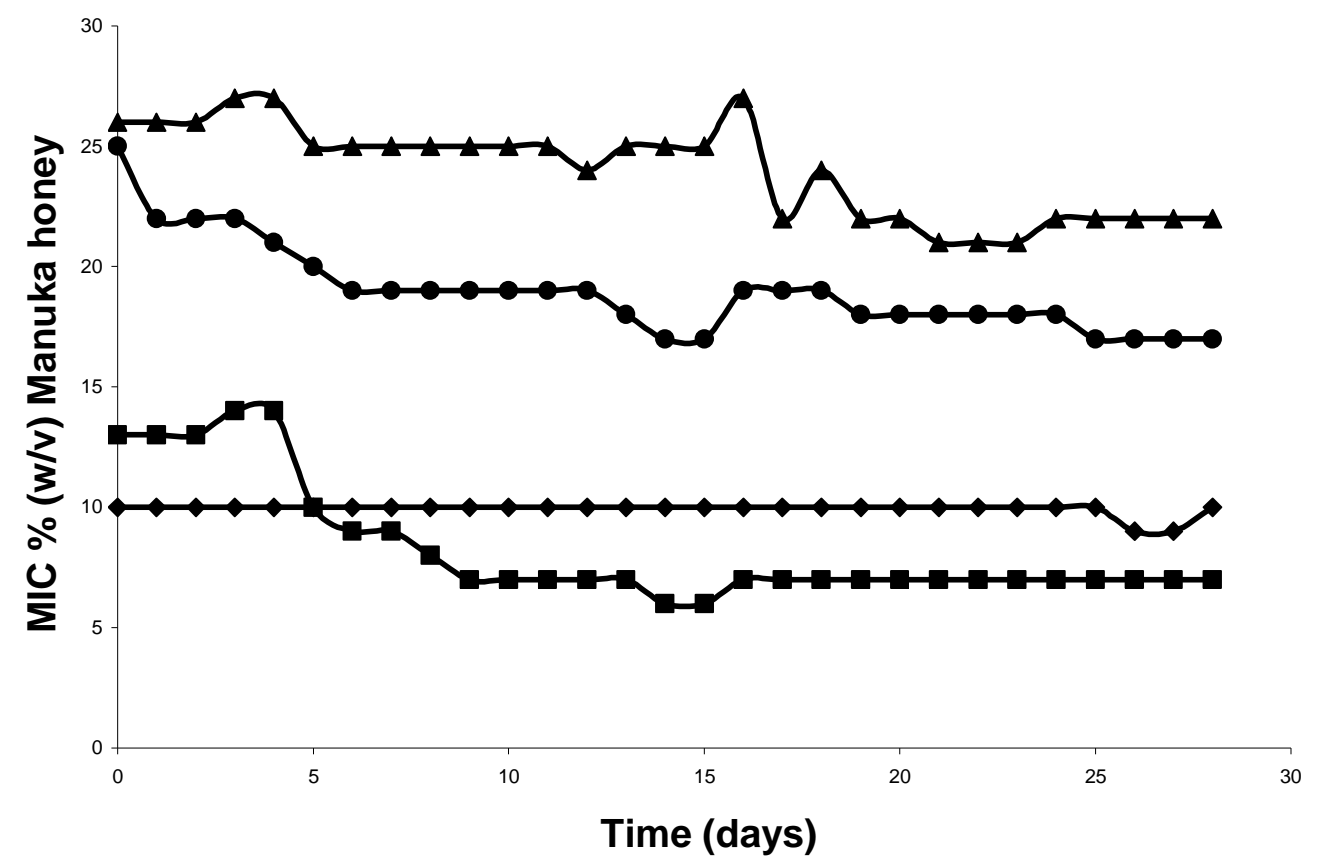

367

368 MICs of manuka honey in subcultures of four 'honey-trained' clinical isolates

369 during a 28 day recovery period in honey-free media: E. coli (triangles),

370 MRSA (diamonds), P. aeruginosa (circles) and S. epidermidis (squares)

371 Table 2: Sensitivity of clinical isolates to manuka honey following long-term

372 exposure, recovery in honey free media and storage at $-80^{\circ} \mathrm{C}$.

\begin{tabular}{|l|c|c|}
\hline Test organism & MIC $^{*}$ & MBC $^{*}$ \\
\hline E. coli & $16.17 \pm 1.47$ & $18.0 \pm 1.67$ \\
\hline MRSA & $5.83 \pm 1.6$ & $8.5 \pm 2.5$ \\
\hline P. aeruginosa & $15.33 \pm 2.25$ & $15.67 \pm 3.2$ \\
\hline S. epidermidis & $5.67 \pm 1.63$ & $8.33 \pm 2.56$ \\
\hline
\end{tabular}

$373{ }^{*}$ Mean manuka honey concentration as $\%(\mathrm{w} / \mathrm{v}) \pm$ standard deviation (tests

$374 \quad$ were done in duplicate on three separate occasions) 